\title{
Zur Echtheit von Cyprians 3. Buch der Testimonia.
}

\author{
Von P. Glaue in Gießen.
}

Mit Arbeiten zur Geschichte des katechetischen Unterrichts beschäftigt, werde ich veranlaßst, auch die Testimonia Cyprians ${ }^{x}$ in Betracht zu ziehen, und da legt es sich mir nahe, von neuem die Aufstellungen betreffend die Echtheit des 3. Buchs zu prüfen, die ich seiner Zeit in meiner nicht veröffentlichten Lizentiatenarbeit gemacht habe. Zugleich ist mir damit Gelegenheit gegeben, Harnacks Ausführungen zu dieser Frage in "Die Chronologie der altchristlichen Literatür bis Eusebius“, 2. Bd. I904, S. 335 u. Anm. zu untersuchen. Ich mub bekennen, daß sich für mich trotz seiner Bemerkungen die Wagschale immer noch zu Ungunsten der Echtheit von Test. III senkt. Während $H$. früher in seiner Schrift "Über den pseudocyprianischen Traktat de aleatoribus" gelegentlich die Behauptung aufgestellt hatte, "daß der cyprianische $U_{r}$ sprung des 3. Buches der Testimonien gewissen Bedenken unterliege", sagt er in der Chronologie: "ein wiederholtes Studium der Frage hat mir die wichtigsten Bedenken niedergeschlagen." Und gerade meine Promotionsschrift habe ihm gezeigt, „daß die Gründe, mit denen man die Echtheit bestreiten kann, nicht durchschlagend sind." Es handelt sich hier um eine verschiedene Bewertung des Für und Wider; eine Einigung wird, glaube ich, kaum zustande kommen. Aber ich möchte doch nicht unterlassen, unter Berücksichtigung der von $H$. angeführten Einwände meine auch jetzt.noch abweichende Stellung in dieser Frage anzugeben.

Mit Recht bestreitet auch $\mathrm{H}$., ohne indes länger dabei zu verweilen, die Beweiskraft des Aufsatzes von J. Haußleiter in den Commentationes Wölfflinianae I89I "Cyprian-Studien" S. 377 ff., der sich für die Echtheit

× Diese - mindestens die beiden ersten Bûcher - gehören meiner Meinung nach zur katechetischen Literatur der alten Kirche; gegen $\mathrm{H}$. J. Holtzmann „Die Katechese der alten Kirche" in Theol. Abhandlungen C. von Weizsäcker gewidmet 1892, S. 107.

28. เo. 1907 . 
von Test. III eingesetzt hatte. Es ist aber doch notwendig, ausführlicher auf ihn einzugehen; prüfe ich seine Ausfuhhrungen, so fält zunächst die Unterstützung fort, die er daraus für seine These der Echtheit erhoffte, zum andern ergibt sich daraus vielmehr ein Grund zu Ungunsten der Echtheit. Einmal nämlich glaubte Haußleiter die überraschende Angabe im Indiculum von 359, das 3. Buch habe nur 770 Stichen, auf einen kleinen Schreibfehler zurückführen und mit Richtigstellung desselben die Echtheit von Test. III stützen zu können. Die Anm. 12 auf S. 385 handelt davon. $\mathrm{Da}$ nach der von ihm aufgestellten Proportion 525 (Zeilen in Hartels Ausgabe): 550 (Stichen im Indiculum) $=1690$ (Zeilen bei Hartel): $\mathrm{x}$ (Stichen) sich für das 3. Buch eine Anzahl von 1770 Stichen ergibt, so wirft Haußleiter die Frage auf: Ist etwa das Zeichen für Tausend $\infty$ vor DCCLXX ausgefallen? Hält nun Haußleiter die Übereinstimmung der Hunderter und Zehner selbst schon für auffallend, so hätte ihn anderseits die Einfachheit dieser Lösung mißtrauisch machen sollen. Eine kleine Probe auf sein Exempel hätte genügt: hätte er eine Proportion zwischen dem 2. und 3. Buche aufgestellt, so wäre die Rechnung nicht so glatt aufgegangen. Wie steht es aber überhaupt mit seiner Proportion? Sieht man genauer $z u$, so lassen sich in den Angaben Haußleiters Ungenauigkeiten, die das so sehr überraschende Resultat ermöglichten, aufzeigen; damit wird es aber hinfällig.

Man darf sich die Mühe kleinlichen Nachrechnens nicht verdrießen lassen. Zunächst zähle ich im 3. Buch bei Hartel 1869 Zeilen (gegen 1876 bei Haußleiter, wobei wir, selbst wenn I oder 2 IVörter im Hartelschen Text eine neue Zeile bilden, diese als selbständige Zeile gezählt haben). Davon sind nun aber nicht bloß 186 Zeilen abzuziehen, wie es Haußleiter tut, als solche, die nur $\mathrm{W}$ - der abweichende Bibeltext erweist sie als nicht cyprianisch - enthält (cf. S. I34, I5- I38, 2 I, $143,2-14,161,8-163,26$ ), sondern noch dazu 17 , die nur $V$, und 3 , die nur $\mathrm{M}$ und $\mathrm{V}$ bieten. Ziehen wir diese 206 Zeilen von $1869 \mathrm{ab}$, dann erhalten wir die Zeilenanzahl der besten Codd., die die Test. enthalten: 1663. Daß A 29 Zeilen ausgelassen hatte, kommt nicht in Betracht. $V$ und $M$ sind sicherlich nicht besscre Zeugen als $W$ und stehen ihm an Textesabweichungen nicht nach. Ferner aber: wer im 3. Buch die Interpolationen des cod. W nicht mitzählt, muß doch im I. Buch ebenso verfahren: von 518 Zeilen im Ganzen sind 20 abzuziehen, nämlich 9 , die $W, 10$, die $W$ und $V$ bieten, und noch eine, die nur durch $B$ und $\mathrm{V}$ geboten wird. Stelle ich nun die Proportion auf: 498 (Zeilen bei Hartel): 550 (Stichen im Indiculum) $=1663$ (Zeilen bei Hartel): $\mathbf{x}$ (Stichen), 
so crgibt sich als Stichenzahl für das 3. Buch 1837. Dem entspricht auch cine Proportion, die vom 2. Buclie ausgeht. Jedoch ist die Stichenzahl, die der Cheltenham Cod. nennt, 850 falsch, die Zahl muß unbedingt größer sein, als die Zahl der Zeilen bei Hartel. Setzt man aber statt dessen die Zahl ein, die der Sangallensis bietet, 950, so ergibt sich: 870 (die 19 Zeilen, die nur W A M bieten, sind unberücksichtigt gelassen): $950=1663: x$ und hier folgt dann als Stichenzahl für das 3. Buch $x=1816$, also nur um 21 geringer als das 1 . Resultat. Die so übcraus einfache Lösung, dic Haußleiter gefunden hattc und durch die er für die Zeit des Indiculum unser 3. Testimonienbuch in dem Umfange, den wir heute kennen, feststellen zu können meinte, ist aufzugeben. Damit ist die Stütze, die Haubleiter für seinen Beweis der Echtheit beibringt, hinfällig.

Weiter wollte Haußleiter die Echtheit von Test. III daraus erweisen, daß Cyprian Test. III in anderen seiner Schriften benutzt habe; ganz besonders deutlich sei das in de hab. virg. Wie aber verhält es sich mit dieser Beweisführung?

Sieht man von den Schriften quod idola dii non sunt, de spectaculis, de bono pudicitiae, de laude martyrii, ad Novatianum, de aleatoribus, de rebaptismate ab als solchen, die nicht von Cyprian stammen und mit Gewißheit oder nur hypothetisch anderen Schriftstellern zugeschrieben werden, so bleiben neben den Test. noch II Schriften übrig, deren Abfassung durch Cyprian behauptet wird. Unter diesen nimmt die Schrift ad Donatum de gratia dei eine besondere Stellung ein; hier behandelt Cyprian sein Thema "von dem neuen Leben nach der Wiedergeburt", ohne daß er zur Bekräftigung seiner Ausführungen förmlich Bibelzitate beibringt. Die übrigen ro Schriften ad virgines, de exhortatione martyrii (ad Fortunatum), de opere et eleemosynis, de catholicae ecclesiae unitate, de dominica oratione, de mortalitate, de lapsis, de bono patientiae, ad Demetrianum, de zelo et livore zeigen darin eine Übereinstimmung, daß mehr oder weniger biblische Belege die gedankenmäßigen Entwicklungen ablösen und beleben. In den 3 Büchern der Test. - den Übergang dazu bietet die Art, wie ad Fort. abgefaßt ist, - sind dagegen die logischen Begründungen zu kurzen thematischen Sätzen zușammengeschrumpft und den Hauptbestandteil machen die I02 resp. 178 und 455 biblischen Zitate aus, die die einzelnen Thesen belegen. Während aber Test. I und II keine Berührungen mit anderen echten Schriften Cyprians in den Zitaten erkennen lassen, steht Test. III in dieser Beziehung ganz anders da: 172 Zitate, davon 67 a. t. und I05 n.t., aus 
Test. III finden sich auch noch in jenen so Schriften teils einmal, teils häufiger wieder.

Nun erhebt sich die Frage nach der gegenseitigen Benutzung. Hat eine solche stattgefunden und ist, wie Haußleiter will, Test. III die Quelle für die übrigen Schriften gewesen? Beginnen wir mit de hab. virg., jener Schrift, an die auch Hauljeiter seinen Beweis vornehmlich angeknüpft hat. Sie bietet 32 Zitate, II a. t., 2 I n.t., von denen sich 28 mit solchen aus Test. III decken und zwar 7 mit solchen aus Kap. II, je 5 mit solchen aus Kap. 32 und aus Kap. 66; 3 finden wir in Kap. 36 wieder und 2 in Kap. 55, je $I$ in Kap. 10. 19. 27. 58. 64. 92. Jetzt meint Haußleiter: die Übereinstimmung zwischen de hab. virg. c. I und Test. III c. 66 sei auffallend, beidemal werden die gleichen Zitate geboten. Finden wir weiter in Test. III 66 die Überschrift disciplinam dei in ecclesiasticis praeceptis observandam, bemerken wir in dem Satze, mit dem Cyprian in de hab. virg. zur Anwendung überleitet quodsi in scripturis sanctis frequenter et ubique disciplina praecipitur et fundamentum omne religionis ac fidei de observatione ac timore proficiscitur, quid cupidius adpetere .... convenit in den gesperrt gedruckten Worten sprachliche Anklänge, so ergäbe sich, daß de hab. virg. c. I von Test. III 66 abhängig sei. Demgegenüber behaupten wir, aus diesen Anklängen ergibt sich viel leichter das Umgekehrte: der Verfasser von Test. III hatte diese Sätze in de hab. virg. gelesen und bildete nun aus den wichtigsten Begriffen seine These Test. III 66, die er mit denselben Zitaten belegte, die er in de hab. virg. c. I fand. Es wäre doch sonderbar anzunehmen, daß ein Mann wie der Verf. von de hab. virg., der so viele Gedanken zur Ausführung seiner Schrift beizubringen hatte, der fortlaufend seine Ansichten entwickelte, sich aus jener These in Test. III 66 Stoff geholt haben sollte, daß dagegen jemand, der eine Thesensammlung anfertigt, sein Material aus anderen Werken erliest und zusammenstellt, befremdet durchaus nicht. Das ist ein Verfahren, für das man manche Belege beibringen könnte. Und was die Anordnung der Zitate betrifft, so ist im Gegensatz zu Haußleiters Ausführungen unseres Erachtens aus der Reihenfolge in de hab. virg. c. I keineswegs zu erweisen, daß sie gegenüber der in Test. III 66 geändert worden sei. Die ersten 4 Zitate in de hab. virg. c. I sprechen ganz im allgemeinen von der Mahnung "haltet Zucht" und nur das letzte bringt noch eine besondere Ermahnung an die Hirten, die mit Zucht weiden sollen. Das ist doch eine gute Ordnung, die für die Originalität spricht. Leider können wir nicht angeben, aus welchen Gründen man die Reihenfolge 
der Zitate in Test. III 66 anders geboten hat, als in de hab. virg. c. 1, aber Willkiir ist bei der Abfassung von Test. III ja auch sonst nicht ausgeschlossen gewesen, sic mag auch hier im Spiele gewesen sein. Ubrigens steht die Jer.-stelle auch in Ep. Cypr. IV ad Pomponium an erster Stelle.

Eine Zusammenstellung der Test. III mit de exhort. mart. ad Fort. liefert nun aber entsprechende, interessante Ergebnisse. Ad Fort. hat im ganzen 85 Zitate, davon finden sich 43 in Test. III wieder und zwar 15 in Kap. 16, je 5 in Kapp. 11 und 59, 4 in Kap. 10, je 2 in Kapp. 6, $15,17,18$, je 1 in Kapp. 20, 26, 27, 28, 29, 61. Von den 23 Zitaten, die wir in den Schlufkapiteln von ad Fort.: 11, 12, 13 haben, stimmen II mit Zitaten aus Test. III 15. 16. I7 überein, 2 finden sich an anderen Stellen von Test. III ( 10 u. 20), 2 in III $I 7$ unter den nur von V vertretenen Zitaten, 8 lassen sich nicht in den Test. nachweisen. Andererseits sind von den 26 echten Zitaten in Test. III 15. 16. 17 - die nur durch $\mathrm{V}$ vertretenen sind nicht gerechnet -18 aus ad Fort. geholt und zwar II aus Kapp. II, I2, I3. Sehen wir uns die Verwandtschaft beider Schriften genauer an, so läßt sich auch hier, wie ich meine, die Priorität von ad Fort. nicht verkennen. Man bedenke nur: in ad Fort. c. I3 haben wir eine Ausführung von 38 Zeilen über die These plus nos accipere in passionis mercede quam quod hic sustinemus in ipsa passione. Die Christen werden zum Martyrium aufgefordert und es wird ihnen der Lohn in Aussicht gestellt, dessen sie, wenn sie als triumphantes zum Paradies zurückkehren, teilhaftig werden sollen. In diesem $\mathrm{Zu}$ sammenhang führt Cyprian ein Zitat an, Röm 8, 18, in dem Paulus diesem Gedanken Ausdruck gegeben hat. Das macht doch alles unbedingt den Eindruck der Ursprünglichkeit. Ganz anders steht es dagegen mit Test. III 17. Nachdem der Verf. in Test. III 16 über de bono martyrii 21 Zitate zusammengestellt hatte, die er bis auf 5 in ad Fort., namentlich in Kap. 12 vorfand, stellt er in III 17 eine These auf, die in negativer Form die These ad Fort. c. 13 wiedergibt: minora esse quae in saeculo patimur quam sit praemium quod promissum est. Und merkwürdig! Als einzigen Beleg bietet er hierfür auch nur wieder jenes Zitat, das wir in der langen Ausführung ad Fort. c. I3 finden. Die klare und schöne Schrift Cyprians ad Fort., in der er auf alle Fragen, das Martyrium betreffend, eingeht, war in der damaligen Zeil viel gelesen worden und sehr bekannt. Ist es da zu verwundern, wenn ein etwas später lebender Christ, als er eine Sammlung von Verhaltungsmaßregeln für die Christen veranstaltete und dabei auch etwas über das 
Martyrium sagen wollte, dies in Anschluß an Schriften tat, die schon in der Kirche Ansehen genossen? Ebenso steht es mit den Beziehungen zwischen ad Fort. c. I und Test. III 59. Im 5. Kap. der Einleitung zu ad Fort. hat Cyprian seine Thesen zu einer Liste zusammengestellt; er bietet hier die Thesen ausführlicher als in der Abhandlung selbst, wo sie oft nur als ein kurzer Auszug erscheinen. Da handeln dann die Thesen $\mathrm{I}-5$ von den Götzen und der Götzenverehrung, vor denen sich die Christen zu hüten hätten. These I der Liste beginnt.mit den Worten idola deos non esse quae homo sibi faciat, was noch weiter ausgeführt wird. In der Abhandlung heißt die I. These quod idola dii non sint - man erinnere sich der dem Cyprian beigelegten, wohl von Novatian stammenden Schrift gleichen Titels - et quod nec elementa vice deorum colenda sint. Diese Thesen und Ausfuihrungen haben den Verf. von Test. III veranlaßt, in sein Werk auch eine These einzustellen, die da lautet de idolis quae gentiles deos putant. Sehen wir von den nur durch W vertretenen Belegstellen dieser These $a b$, so werden uns hier allerdings in veränderter Reihenfolge 6 Zitate geboten, von denen Cyprian schon 4 für seine I. These ad Fort. beigebracht hatte.

Noch eine Schrift verlangt, daß wir uns ausführlicher mit ihr beschäftigen. Auf 2I Seiten hat Cyprian über de opere et eleemosynis gehandelt und er hat seine Ausführungen mit 4I Zitaten belegt; von diesen finden sich 28 auch in Test. III wieder. Besonders auffallend ist aber folgendes: Der Verf. von Test. III hat an die Spitze seiner Sammlung gestellt de bono operis et misericordiae und in III 2 heißt es in opere et eleemosynis etiamsi per mediocritatem minus fiat ipsam voluntatem satis esse. Während er nun für diese 2. These nur ein einziges Zitat beibringt, belegt er.die I. mit 32 Zitaten. Von diesen finden wir aber 20 in de op. et eleem. vor. Hier scheint uns nun die Priorität von de op. et eleem. unbestreitbar. Im Verlaufe seiner Ausführungen bringt Cyprian nämlich, so wie es ihm gerade nötig erschien, Zitate aus dem AT und NT durcheinander bei; die Auswahl der Zitate erfolgte entsprechend der Gedankenentwicklung. In Test. III sind dagegen die $Z$ itate aus dem AT und NT genau geschieden, die atl. stehen voran; innerhalb beider Gruppen vermissen wir eine strenge Ordnung. Dies Verfahren läßt sich erklären, wenn man annimmt, die Zitatenreihen von de op. et eleem. haben ihm im Zusammenhang der größeren Abhandlung, vorgelegen. Der Verf. hat sie, soweit sie zu seiner These paßten, übernommen, ordnungsgemäl gruppiert und durch einige andere, die ihm beweiskräftig zu sein schienen, ergänzt. Anzunehmen, daß Cyprian 
in einer spätcren Zeit, nachdem cr in Test. III die Belege zu de bono operis et misericordiac schon gegebon hatte, dazu cine weitere Ausführung gemacht habe, bei der einige Zitate nicht verwendet und dafür anderc eingefügt seien, halte ich nicht für ricintig.

Was die Bexiehungen zwischen den übrigen echten Schriften $\mathrm{Cy}$ prians und den Test. III betrifft, so möge es genügen, sie hier in einer Liste zusammengestellt zu haben; dab der Verf. von Test. III sie zum Vorbild gehabt habe, läßt sich nicht erweisen. De cath. eccles. unit. enthält 52 Zitate, 25 davon finden wir in Test. III wieder. $Z$ u bemerken

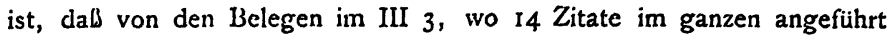
werden, 7 sich auch in de cath. eccl. unit. Kapp. 12, 14, 24, 25 finden und daß 4, die sich in de cath. eccles. un. in Kapp. 6 und 8 finden, in III 86 (mit 6 Zitaten) angeführt werden. De dom. orat. enthält 69 Zitate, 23 davon finden wir in Test. III wieder. $Z u$ bemerken ist, daß von den 6 Belegen in III 56 drei in de dom. orat. c. 4 nachgewiesen werden können, dals 4 Zitate, die in de dom. orat. c. 7 und 14 (hier 3) beigebracht sind, sich auch in III 19 (mit 6 Zitaten) wiederfinden. Höher dem Prozentsatze der wiedergebrauchten Zitate nach steht die Schrift de mort., von deren 27 Zitaten 20 auch in Test. III zum Belege dienen; für Test. III 58, nit 13 Zitaten, haben 7 Verwendung gefunden, die aus de mort. c. 3. 7. 21. 22. 23 (alle 3, die hier stehen) übernommen sind. Für III 6, mit II Zitaten, haben 5 Verivendung gefunden, die aus de mort. c. IO. II. (I2 hat keine Zitate) I3 übernommen sind. Für III I4, mit 6 Zitaten, haben 3 Verwendung gefunden, die aus de mort. c. Io. und I I ubernommen sind. De lapsis hat $4 \mathrm{I} Z$ itate, davon stehen 18 auch in Test. III; in III 56 mit 6 Zitaten finden wir 3 aus de laps. c. 27. De bono pat. hat 32 Zitate, davon stehen 15 in Test. III. Ad Demet. hat 26 Zitate, davon stehen 7 in Test. III; die 2 Zitate in III 50 stehen auch in ad - Demet. c. I. De zelo et liv. hat 20 Zitate, davon stehen 7 in Test. III.

Zum Abschlub dieser Ausführungen möchten wir nun im Gegensatz zu Haußleiter, der Test. III die Priorität zuspricht, behaupten: die Berührungen in den Zitaten zwișchen Test. III und de hab. virg. erklären. sich ebenso wie die Berührungen zwischen Test. III und den beiden Schriften ad Fort. und de op. et eleem. aus der Benutzung dieser 3 Schriften bei der Zusammenstellung von Test. III; die Berührungen zwischen Test. III und den anderen echten cyprianischen Schriften können wenigsten ebensogut so erklärt werden. Das umgekehrte Verhältnis braucht man keineswegs anzunehmen. Haben wir aber mit diesem Resultate Recht, dann ist damit auch hinfällig geworden, was die Ver- 
teidiger der Echtheit von Test. III bisher immer behauptet haben: Test. III sei 1-2 Jalıre nach Test. I und II verfaßt worden. Auf diese Weise wollten sie ja das Vorhandensein der 2. Vorrede bei der Fortsetzung des einmal begonnenen Werkes rechtfertigen, dessen Abfassung man andererseits aber auch nicht viel tiefer hinab - in Cyprians Bischofszeit - verlegen konnte. Nein: hat Cyprian in Test. III, die in seinen anderen Schriften zusammengestellten Konvolute von Bibelstellen verwendet, dann fällt die Abfassung von Test. III - vorausgesetzt eben, daß auch dieses Buch Cyprian angehört, in die späteren Lebensjahre des Bischofs. Mit diesem Ergebnis wird sich dann auch Harnack einverstanden erklären.

Nun halte ich es aber nicht für angängig, dem Schriftsteller und Bischof Cyprian solche Arbeit, wie es Test. III ist, zuzutrauen, auch nicht, wenn ich es wegen der besonderen Vorrede betrachte als „ein Spruchbuch für sich, welches an das zweigeteilte erste Spruchbuch herangerückt worden ist, weil es ihm formell gleichartig ist" (Harnack). $\mathrm{Zu}$ dieser Zusammenstellung eines Spruchbuches zum Bibelersatz hätte sich der viel beschäftigte, in verschiedenen Fehden stark engagierte Bischof wohl kaum die Zeit genommen.

Wie will man es weiter erklären, daß von jenen Kämpfen über die Einheit der Kirche, über die lapsi, gegen Felicissimus, gegen Novatus und gegen Novatian nur ganz wenige Thesen handeln und dies in einer Weise, da@ man ihnen nicht anmerkt, ihr Schreiber habe mitten im Kampfe gestanden. Hätte Cyprian in seinen letzten Lebensjahren Test. III verfa@t, er hätte sicherlich energischer, lebhafter und ausführlicher darüber geschrieben.

Und dann: in Test. III ist eine Menge von I20 Thesen ganz ohne Ordnung, ohne alle Subdivisionen niedergeschrieben. Dogmatische, kirchlich-rechtliche, sozial-ethische Thesen sind so wild durcheinandergewürfelt, daß man erst durch vollkommene Unstellung aller Thesen ${ }^{x}$ eine einigermaßen annehmbare Disposition erhält; jeder größlere leitende Gesichtspunkt, unter den diese Fülle von Thesen gebracht werden könnte, fehlt hier absolut. Sollte ein klarer, genau disponierender Kopf, wie Cyprian es war, wirklich dergleichen gemacht haben? Diese schlechte Disposition von Test. III ist aber um so auffallender, als doch das Ziel, das diese Arbeit im Auge hatte, das war, ad fovendam memoriam zu dienen. Von den beiden ersten Büchern der Test. kann

2 Eine solche biete ich am Schluß. 
man das wohl sagen; da bestcht $z$ Recht, was dic pracf. I aussagt: subtiliore compendio id quod legitur tenax memoria custodit. Wer aber wollte erwarten, daß ein Christ diese disiecta membra von Test. III im Gedächtnis festhielt!

Schen wir uns aber die Vorrede zu Test. III noch genauer an. Stammt sie wirklich von Cyprian, so ist es doch, selbst wenn einige Jahre seit Abfassung jener Bibclzitaten-Zusammenstellung vergangen waren, die dem gleichen Adressaten gewidmet und in ihrer Anlage der neuen Arbeit durchaus ähnlich war, höchst verwunderlich, daß Cyprian in ihr, mochte Test. III auch nicht als 3. Buch gedacht sein, mit keinem Worte jenes vorangegangenen Werkes Erwähnung tut. $\mathrm{Zu}$ aller Zeit hätte man doch in solchem Falle an das bereits Geschriebene mit irgend einer Wendung angeknüpft. Oder will man zu der Ausflucht greifen und dem Cyprian zutrauen, er habe jene Arbeit nicht mehr in der Erinnerung gehabt, er habe sie durch seine Wirksamkeit als Bischof vollkommen vergessen? Nein, so gedächtnisschwach ist Cyprian nicht gewesen. Und zudem läßt sich m. E. zeigen, daß der Verf. bei Abfassung der 2. praef. die I. vor Augen gehabt habe. Bringt sie doch weitaus kürzer und in einer Form, die gegenüber der Vorlage, der I. praef., blaß und abgeschwächt erscheint, nur wenige Gedanken, während sie mit vielen Worten angibt, in welcher Weise der Verf. die Bitte des Quirinus erfüllt habe, woraus selbst Verteidiger der Echtheit von Test. III geschlossen haben, die 2. praef. mit dieser Angabe des Zwecks der Test. sei neben der ausführlichen 1. praef. überflüssig. Darum: Wäre die 2. praef. von Cyprian, er hätte sicherlich an Test. I und II irgendwie erinnert. Ein Mann aber, der seine mit Hilfe cyprianischer Schriften zusammengestellte, den cyprianischen ersten beiden Testimonienbüchern nachgebildete Arbeit herausgab, getraute sich doch nicht, soweit .zu gehen, daß er seine Schrift durch direkten Hinweis auf Test. I und II unter Cyprians Namen stellte; deswegen unterließ er jede Erwähnung des Zusammenhanges mit Test. I und II.

Nun wendet Harnack ein: Das 3. Buch findet sich schon im. Mommsenschen Verzeichnis. 'Er kommt damit auf den Gegengrund zurück, den Haußleiter gegen ihn anführte, als er s. $Z$ t. darauf hingewiesen hatte, daß die Überlieferungsgeschichte der Echtheit des 3. Buchs nicht durchweg günstig sei. Aber ich kann die Berechtigung dieser Gegeninstanz nicht anerkennen. Auch ich meine: in dem Jahrhundert nach Cyprians Tode, also bis zu dem von Mommsen mitgeteilten Indiculum Cecili Cipriani aus dem Jahre 359, ist das 3. Buch 
der Test. in der Form der beiden ersten verfabt und wegen der äußeren Ähnlichkeit mit den beiden anderen an sie herangeschoben worden. Kein Wunder, daß es vielfach, so auch vom Verfasser des Indiculum, dem berühmten Cyprian zugeschrieben wurde. Es mub doch aber seinen Grund gehabt haben, daß die Überlieferungsgeschichte sich häufig gegen den cyprianischen Ursprung von Test. III wandte, und ich sehe nicht ein, wie ich mir die Mißgunst derselben gegenüber Test. III, wenn sie von andern Bedenken gestuitzt wird, anders erklären soll als dadurch, daß man es eben als unecht beanstandet hat.

Ich wei $§$ wohl, daß auch gegen Test. I und II aus der Überlieferungsgeschichte das Urteil auf Unechtheit gefällt werden könnte. Abgesehen von den Codices, die überhaupt nur einzelne der 14 von Hartel im I. Bande vereinigten Traktate, darunter auch nicht Test., enthalten wie F, O, D, Paris. I655, gibt es ja Codd. oder Verzeichnisse, die alle (oder wenigstens eine größere Anzahl der) Traktate Cyprians, aber die Bücher der Test. nicht bieten wie die Vita per Pontium, nicht lange nach $\mathrm{Cy}$ prians Tode geschrieben, der S(eguerianus) sec. VI-VII, der W(irceburgensis). sec. VIII vel IX, der cod. der Reichenauer Bibliothek, die Augsburger Handschrift (Katalog No. 65), J (Paris I446I) sec. XII, - (Paris I7350) sec. XII, G (Sangallensis) sec. IX, so daß wir schließen müssen, man habe die Test. von der Aufnahme absichtlich ausgeschlossen. Doch da sonst nichts gegen die Echtheit von Test. I und II eingewendet werden kann, so darf auch ihr Fehlen in einzelnen, ob auch z. T. sehr wertvollen Handschriften nicht ausschlaggebend sein; vielleicht haben sie auch unter der Mißgunst gegen Test. III mitleiden müssen, so daß dann diese Handschriftenreihe besonders stark gegen Test. III zeugen würde.

Bei Test. III liegt die Sache aber anders. Hier sind schon unsere Bedenken gegen die Echtheit vorhanden und diese werden nun ohne Zweifel verstärkt, wenn die Überlieferungsgeschichte neues Material dazu liefert. Das ist aber der Fall. Fehlt auch zurzeit leider noch ein statistisch genauer Nachweis darüber, wie groß die Zahl solcher CyprianHandschriften ist, die nur Test. I und II bieten, so haben wir wenigstens einige bestimmte Angaben. i sec. XII-XIII, $\rho$ sec. XII-XIII, Paris. I654 sec. XII-XIII, Paris. 1922 sec. XIII enthalten Test. III nicht. Baluzius sagt: von 2I Handschriften, die er. durchgesehen habe, hätten 5 nur die Test. I und II gehabt. Eventuell ist hierfür auch die nicht ganz verständliche Angabe Turners in Studia Bibl. et Eccles. Oxf. T. III p. 309 auf Grund von Becker über Bobbio catal. sec. X heranzuziehen, 
dab sich daselbst unter No. 152, 153 ad Quirinum libri II finden. Diesen Handschriften füge ich Zeugnisse der Editionen an. Die Editio Spirensis Venedig 1471, die sog. Vetus Veneta, sine nomine Typographi aut Oppidi (soweit crsichtlich bald nach Erfindung des Buchdrucks entstanden) und die editio Remboldi Paris 1512 enthalten nur Test. I und II; Männer wie Rigaltius, Scultetus, Andreas Masius haben immer wieder Zweifel an der Echtheit von Test. III geäußert. Vor allem aber ist hier Erasnus zu nennen, der in seiner 1520 in Basel erschienenen, später noch mehrmals wiederholten Ausgabe cyprianischer Schriften, leider ohne seine Gründe zu nennen, behauptet: das 3. Buch der Test. stamme nicht von Cyprian. Das sei um so wunderbarer, sagt Pamelius, praesertim cum fateatur stilum eius esse, ein Urteil, das Erasmus dann nur aus dem der cyprianischen I. praefatio nachgebildeten Vorwort zu Test. III gewonnen haben kann. $\mathrm{Zu}$ erwähnen ist aber, daß Erasmus in seinen annotationes in evang. Joh. Test. III 53 als cyprianisch zitiert.

Auch das letzte Argument Harnacks macht mich in meiner Ansicht betr. Unechtheit von Test. III nicht irre. Er schreibt: „Dab dieselbe Bibeltext-Rezension wie in Test. I und II und den anderen Cyprianschriften so auch in Test. III benutzt ist, ist gegenüber Bezweiflung ihrer Echtheit von Wichtigkeit." $\mathrm{Da}$ ich auf dem Standpunkt stehe, daf man die Abfassung von Test. III nicht allzulange nach Cyprians Tode ansetzen soll, zu einer Zeit also, als man seine Bibelzitaten-Sammlung Test. I und II in den karthagischen Gemeinden noch benutzte und nur einer Etgänzung derselben bedurfte, da ich auch annehme, daß dieselbe karthagischen Ursprungs ist, so halte ich es für durchaus möglich, daß ihr Verfasser für seine Zitate den Bibeltext benutzte, den auch Cyprian verwendet hatte. Es war eben der in Karthago gebräuchliche Bibeltext, wie er nach Tertullians Zeit festgestellt worden war.

Nun möchte ich hier noch eine Hypothese über den Verfasser der Test. III vortragen. Ich wage es, trotzdem mein Zutrauen zu ihr schwach geworden ist, in dem Gedanken, daß vielleicht ein anderer mit Hilfe dieser Andeutungen zur Lösung vorliegender Frage einen richtigeren. Weg findet, als es mir möglich ist.

I. Nach obigen Ausführungen gilt bezüglich des Terminus der Abfassung von Test. III, daß sie nach Cyprians Tode, aber wegen des soeben angeführten Argumentes nicht allzulange nach demselben entstanden sind.

2. Es liegt keine Veranlassung vor, an den Nachweisen zu zweifeln, die Dombart Z. w. Th. I879 geliefert hat. Danach zeigen 
Commodians Carmen apologeticum und Instructiones Berührungen mit den Testimonien und zwar auch - dies gilt vor allem für Instructiones mit Test. III.

Wir sagen nun: I. Führt uns die Bestimmung der Lebenszeit Commodians auch auf die Zeit bald nach Cyprians Tod, so können Test. III von Commodian stammen. Über die Lebenszeit Commodians sind allerdings die Nachrichten, die wir haben, spärlich und unbestimmt cf. hierzu Dombarts Artikel "Commodianus"! in HRE3 IV 250ff.: a) hat man Commodian in die Zeit Cyprians versetzt, ${ }^{x}$ so wurde man dazu durch die Berührungen veranlaßt, die man zwischen Commodians Werken und den als echt angenommenen und darum in die Jahre $248-250$ angesetzten Testimonien aufdeckte. Ist nun aber Test. III nicht cyprianisch, ja nachcyprianisch, dann fällt mindestens auch die Abfassung von Instructiones - will man nicht $z u$ der Annahme greifen, die Instructiones seien das Primäre - nach Cyprians Tod. b) wenn Ebert (Abh. der Kgl. Sächs. Ges. d. W. Leipzig 1870) und Krüger (Altchristl. Litt. \$89, 36) vor allem 2. Punkte anführen, die die Absicht, Carmen apologeticum sei für das Jahr 249 festgelegt, stützen sollen: den Hinweis auf die bevorstehende Verfolgung - das sei die decianische - und den Übergang der Goten über die Donau, der 249/250 stattgefunden habe, so ist das als Bewveis für die Abfassung von Carm. apol. und in weiteren damit als Angabe für die Lebenszeit Commodians hinfällig. Wer will denn beweisen, dalj unter der bevorstehenden Verfolgung die decianische gemeint ist? Auch wenn Commodian sie als die 7 . Verfolgung bezeichnet, so weiß man doch nicht, welcher Zählung er folgt. Die, der auch Augustin in de civ. Dei lib. XVIII c. 52 folgt, soll die gewöhnliche gewesen sein und deshalb soll Commodian sich nach ihr gerichtet haben (so Ebert). Ja, ist denn aber die Zahl 7 nicht als apokalyptische Zahl geläufig genug gewesen, so daß der Dichter, ohne überhaupt nachgerechnet zu haben, sagen kann, „die 7. Verfolgung wird den Anfang der letzten Dinge machen?“ Der Goteneinfalle sind es auch mehrere gewesen. .Dazu komint, daß die Nachrichten der Quellen schwankend und widersprechend sind, wie Ebert selbst zugesteht. So versieht denn auch schon Dombart HRE 3 IV 252 das Jahr 249 mit einem Fragezeichen.

Ẹs hindert uns also nichts, mit dem Ansatz für die Lebenszeit

I cf. Dombart, HRE3 IV 251, 20 "Commodians Gedichte wurden um die Mitte des 3. Jahrhunderts verfaßt." Krüger, Altchristl. Litteratur S 89 "Commodian scheint um die Mitte des 3. Jahrhụnderts gewirkt zu haben." 
Commodians hinter den Tod Cyprians hinabzugehen. Wir möchten uns zu Harnacks Bcstimmung LG S. 73I bekennen: nach Cyprian und vor Theodosius I, wahrschcinlich unter Valerian oder Diocletian.

2. Wir nahmen an, daß Test. III in Afrika verfalt worden ist. Nun haben wir zwar über das Geburtsland Commodiaris keine bestimmte, zuverlässige Angabe. Aber nach mancher seiner romfeindlichen Äuberungen wäre Afrika als Aufenthaltsort für seine Mannesjahre wenigstens (cf. Dombart HRE) recht gut anzunehmen. Also auch hiernach kann Commodian Test. III geschrieben haben.

3. Erweist sich unsere Hypothese, Commodian ist der Verfasser von Test. III, als richtig, so ist es leicht verständlich, wie es zu der Benutzung von Test. III in den Instr. kam: seine Zusammenstellungen der Thesen und biblischen Zitate verwendet er später, als er gleiche Werke in Gedichtform verfaßte. Dadurch erklärt es sich vielleicht auch, daß seine Verse so wenig gut sind, wenn wir daneben auch in Betracht ziehen müssen, daß er sich absichtlich einer ungefeilten, dem Volke angemessenen Sprache bediente. Daf Commodian für seine Dichtungen auch Test. I und II benutzt hat - cf. Carm. apol. ${ }^{x}-$ ist nicht dadurch ausgeschlossen, dals die cyprianische Abfassung von Test. I und II aufrecht erhalten wird. Hat er Test. I und II doch wohl schon bei der Abfassung seiner Testimoniensammlung (Test. III) als Vorlage gebraucht.

4. Ohne kirchliche Stellung - die handschriftliche Überlieferung, die ihn als Bischof bezeichnet, ist wohl falsch - aber populär-theologisch interessiert, wollte er durch seine Arbeiten dem Volke dienen, ihm die Kirchenlehren und Kirchenvorschriften, sowie die Bibel bequem zugänglich zu machen. Diese Absicht suchte er im Anschluß an Test. I und II vielleicht zunächst durch Test. III zu erreichen. Dann aber hielt èr es für noch wirkungsvoller, wenn er durch populäre Dichtungen an das Volk herantrat. $\mathrm{Ob}$ deshalb vielleicht Test. III nicht vollendet wurde und sich so seine mangelhafte Disposition erklärt? Die 2. praef. braucht nicht von Commodian zu stammen. Vielleicht wollte ein Späterer durch diese zusammengestückelte praef. Cyprians Sammlungen (Test. I und II) und Commodians (nicht vollendete?) Arbeit verbinden und letztere - als Bibelersatz gut brauchbar - auch unter Cyprians Namen verbreiten.

I Die Zitate aus III 59, die sich in Carm. apol. wiederfinden, werden nur durch IV geboten. 
5. Das Mißtrauen, das Test. III gegenüber gewaltet hat und das auch auf Test. I und II übertragen wurde, wird jetzt erklärlich: Commodian galt als heterodoxer Christ, er war Patripassianer und Chiliast - seine Gedichte gelten noch im sog. Decretum Gelasianum als apokryph s. Harnack. Pontius und der Schreiber der Vorlage des Seguerianus ich nenne sie als die ältesten Zeugen - nahmen Test. I und II nicht auf, weil sie infolge der Verbindung derselben mit Test. III - cf. die gleiche Anlage und an denselben Adressaten gerichtet - als dessen Verfasser sie vielleicht noch Commodian kannten, an der cyprianischen Abfassung der Test. I und II Zweifel hegten. Erst später - schon zur Zeit des Indiculum Mommsens aus d. J. 359, in dem die Test., allerdings noch am Ende der Traktate, stehen, läßt es sich konstatieren überwog dann die Autorität Cyprians, der als der Verfasser von Test. I und II bezeichnet wurde, das Mißtrauen gegen Commodian, ja es wird dann auch Test. III - vielleicht ist zu diesem Zwecke die 2. praef. verfalit worden - dem Cyprian zugeschrieben.

6. Inhaltlich schließt sich das Carm. apol. mit seiner dogmatischen Abzweckung an Test. I und II an, Instr. an Test. III. Diese beiden letzten Schriften haben „,vorwiegend praktisch-ethischen Charakter", nur die I. Hälfte des I. Buches der Instr. macht davon eine Ausnahme, wie wir solche Ausnahme ja auch in Test. III finden können - cf. hier 36 dogmatische Thesen. Daß Commodian in den Instr. die Schrift Cyprians de hab. virg. viel benutzt hat (s. Dombart, $Z$. w. Th.), ist nicht verwunderlich, wenn wir nachweisen können, da $\$$ auch Test. III in einem Teil im engsten Anschluß an de hab. virg. entstanden ist.

7. „Das Streben, die Übersicht über die abgehandelten Themata durch Überschriften zu erleichtern", das, wie Dombart schreibt, den Instr. mit den Test. gemeinsam ist, erklärt sich am einfachsten daraus, dab Commodian nach dem Muster Cyprians (Test. I und II) - Thesen belegt durch Bibelstellen - seine Sammlung Test. III angefertigt und diese eigenartige Ausführung dann auch in den Instr. beibehalten hat. Hei@t es..dann aber weiter: „Außerdem zeigt sich auch im einzelnen häufig eine solche Übereinstimmung der Instr. hauptsächlich mit dem 3. Buch der Test., daß eine Abhängigkeit Commodians von Cyprian auch hier angenommen werden muß (Instr. II $16=$ Test. III I I, Instr. II 30 Überschrift $=$ Test. III 109 Überschrift. .Instr. II $30,12=$ Test. III 96 Überschrift, Instr. II 5 Überschrift $=$ Test. III 98 Überschrift, Instr. I $4 \mathrm{I}$ = Test. III II8 Überschrift)“, so scheint diese Übereinstimmung für Dombart doch etwas Auffallendes zu haben. Nimmt man jedoch für 
Test. III und Instr. densclben Verfasser an und läbt man dic Abhängigkeit von Cyprian ganz beiseite, so versteht man recht wohl, wie es zu dieser Übereinstimımung kommt. Zuletzt ethält dann auch „der Umstand, daß weitaus der grölite Teil von Commodians Bibelzitaten und biblischen Anklängen sich auch in den Test. findet", seine gute Erklärung.

8. Dürte man nun soweit gehen und die Annahme wagen, Commodian habe den Vornamen Quirinus geführt - wir wissen sonst nichts über den Vornamen des Commodian und auch der Adressat der Test. ist uns sonst unbekannt - so kämen wir zu folgendem, kühnem Resultat: Cyprian schrieb Test. I und II auf Bitten des Commodian, den er getauft hatte - daß Quirinus nicht als Christ geboren war, wobei es unentschieden bleibt, ob er von Haus aus Heide oder Jude war, haben wir a. a. O. zu zeigen versucht-; Cyprian widmete ihm diese Ausführungen (quibus non tam tractasse quam tractantibus materiam praebuisse videamur) und Commodian arbeitete nun, aufgefordert durch die Anregung, die er erhalten hatte (cf. I. praef.: bibere uberius et saturari copiosius poteris, si tu quoque ad eosdem divinae plenitudinis tontes nobiscum pariter potaturus accesseris) in der gleichen Weise weiter, stellte das 3. Buch nach dem Muster der beiden. ersten zusammen - ob es fertig war, ist fraglich s. o. - und arbeitete nun, um dem Volke zu dienen, das $\mathrm{zu}$ Gedichten um, was ihm gewidmet worden war und was er selbst ausgeführt hatte.

Disposition zu der Thesensammlung in Test. III.

I. Abteilung. Dogmatische Thesen.

A. Die Personen der Gottheit.

I. Gott (Liebe zu Gott, Gottesfurcht, Monotheismus, durch Christus zu Gott): These I8. 19. 20. 35. 56. 100. 55. 59. 24.

2. Christus (Verehrung Christi, Leben in Christo): These 33. 39 (I8. 24).

3. Heiliger Geist (keine Süünde wider ihn, Erscheinungen desselben): These 7. ror.

B. Die beiden Reiche.

r. Reich Gottes. a) Fundament desselben, b) Gesetz und Evangelium, c) über den Glauben: These a) 69; b) I 19; c) I0. 3 I. 42. 45.52.

2. Reich der Welt. a) Die Sünde als herrschendes Prinzip, b) der Teufel als Herrscher, c) Weltgericht: These a) 6. 15. 38. 47. 54. 57. 79. 91. 98; b) 80. II7. II8; c) 89. 99. II2. 
2. Abteilung. Kirchliche Thesen.

A. Ausführungen über die Sakramente a) im allgemeinen und b) im einzelnen (1. Abendmahl, 2. Taufe, 3. Buße): These a) $53 ; b_{1}$ ) 50 . $\left.\left.94 ; b_{2}\right) 25.26 .27 .43 .65 .97,116 ; b_{3}\right) 28$. I II. II 4 .

B. Kirchliche Anweisungen zum rechten Verhalten als Glied der Kirche:

I. gegenüber der heidnischen Religion Aufrechterhalten der Einheit: These 93. 86 .

2. Aufrechterhalten der kirchlichen Zucht a) in der Gemeinde, b) gegen die Oberen: These a) 46. 66. 67. 68. 77. 78. 95 ; b) 85 .

3. Erfüllung spezieller kirchlicher Vorschriften (Gebote, Verbote): These 30. 120; 12. 82 .

C. Ausfuhrungen über das Leiden, speziell das Martyrium (dessen Ursachen und Segen): These 14; 29. 37; 16. 17. 58.

3. Abteilung. Individual- und sozial-ethische Thesen.

A. Über den rechten persönlichen Lebenswandel

1. allgemein: als Christ im Gegensatz zum Heidentum: These 34. 64. 11 .

2. speziellere Angaben über die Lebensführung des Christen (Bescheidenheit im Sprechen und im Handeln): These 4I. 103; 92. 96. 4. 5. 51. 102; 60. 61.

3. rein äußerliches Verhalten: These 36.83 .84 .

B. Über den rechten Wandel in der Gemeinschaft

I. im Haus a) Ehe, b) Familie, c) Dienerschaft: These a) 32.62. 63. 90 ; b) 70. 71 ; c) 72. 73. 105 .

2. in der weiteren christlichen Gemeinschaft a) Gebot der Förderung des Nächsten durch Unterstützung, b) Warnung vor Schädigung des Nächsten: These a) I. 2. 3. 9. 40. 75; 74 . 109. II 3; 49. 22. 23. 106. 44. 76; b) 13. 21 . 104. 107. I10. II5; 8. $88.108 ; 48.8 \mathrm{I}$. 